\title{
Subsurface imaging: a new engineering discipline
}

\section{Bahaa Saleh}

Bahaa E. A. Saleh, "Subsurface imaging: a new engineering discipline," Proc. SPIE 9663, Eighth International Topical Meeting on Education and Training in Optics and Photonics, 96630T (6 October 2003); doi: 10.1117/12.2208498

SPIE Event: Eighth International Topical Meeting on Education and Training in Optics and Photonics, 2003, Tucson, Arizona, United States 


\title{
Subsurface imaging: a new engineering discipline
}

\author{
Bahaa E. A. Saleh \\ Department of Electrical and Computer Engineering, and Center for Subsurface Sensing and Imaging Systems, Boston University \\ 8 Saint Mary's Street, Boston, MA 02215 \\ Phone: (617) 353-7176,fax(617) 353-6440,e-mail: besaleh@bu.edu
}

\begin{abstract}
Subsurface imaging is a new discipline encompassing optical, electromagnetic, and ultrasonic tools for underwater, underground, or under tissue imaging. We have developed a new senior-level engineering course covering waves and their interaction with various media, inverse scattering methods, and image reconstruction techniques for localized and multiview tomographic imaging.

(C)2003 Optical Society of America

OCIS codes: (000.2060) Education
\end{abstract}

\section{Introduction}

Subsurface Imaging is a new discipline encompassing optical, $\mathrm{x}$-ray, electromagnetic, electrical, and ultrasonic methods for imaging objects that are hidden under water, under ground, under tissue, or below man-made objects such as layers of fabricated material. Despite the broad medical, biological, geophysical, environmental, and industrial applications, and the wide range of technologies involved, the underlying fundamental principles and methodologies are rather similar and can be taught as a single unified discipline.

A course covering such discipline has been pioneered by the Center for Subsurface Sensing and Imaging Systems (CenSSIS), an Engineering Research Center (ERC) funded by the National Science Foundation since 2000. The new course has been developed, in various forms, at the four universities that make the CenSSIS academic partnership (Boston University, Northeastern University, University of Puerto Rico at Mayagüez, and Rensselaer Polytechnic Institute), and is being infused into their engineering curricula.

\section{Subsurface imaging course}

The course that I have developed at Boston University and taught in the Spring of 2003 is a senior, or first-year graduate, multi-disciplinary engineering course available to students with an interest in fields and waves, signal and processing, biophotonics, and/or ultrasonics. Topics include: waves and their interaction with various media, 2D and 3D imaging techniques, image reconstruction from tomographic projections, and inverse problems.

The following is a brief description of the course content.

1)An introduction to waves compares and contrasts electromagnetic and acoustic waves and covers phenomena such as reflection, refraction, diffraction, and scattering. A brief study of scattering in random media leads to diffuse wave optics. Both $\mathrm{CW}$ and pulsed waves are discussed, and the role of dispersion is highlighted.

2) 2D Image formation for both scanning and gazing systems is covered in the context of microscopy. The concept of point spread function and modulation transfer function is presented for both coherent and incoherent imaging, and resolution limits are highlighted. This is followed by an introduction to the imaging of phase objects.

3)Methods of $3 \mathrm{D}$ imaging include axial focusing and confocal systems; time of flight sectioning, which is the basis of radar and sonar methods; and interferometric techniques in the time, frequency, and coherence-domains.

4) The course subsequently moves to the Image reconstruction and processing aspects of subsurface imaging. This section of the course begins with methods for deblurring linearly blurred images, for both 2D and 3D systems with known point spread functions. Algebraic techniques such as singular value decomposition are covered.

5)We then turn to reconstruction from projections by use of techniques such as filtered back projection. Applications include computerized tomography and MRI. Inverse source problems and inverse scattering problems are then addressed under simplifying assumptions (Born approximation), and applications to diffraction tomography are highlighted, emphasizing the technique of filtered back propagation.

6) The course ends with the more difficult reconstruction problems for which linearization methods are not appropriate. This includes numerical iterative techniques applicable to problems such as ground penetrating radar, diffraction tomography, diffuse optical tomography, and electrical impedance tomography.

One of the requirements of the course is a paper or presentation covering one of the applications of subsurface imaging in some detail. The material is to be based on first-hand observation of projects in a laboratory setting. This is not difficult in the environment of a large multi-university center such as CenSSIS. 